\title{
Serological Survey of Crimean-Congo Hemorrhagic Fever Virus Kukes and Has, Albania
}

\author{
Përparim Kadriaj \\ Majlinda Dhimolea-Kota \\ Enkelejda Velo \\ Silva Bino
}

Infectious Disease Control Department, Institute of Public Health, Tirana, Albania

\begin{abstract}
In Albania the first cases of Crimean-Congo hemorrhagic fever was identified in 1986. In Albania, cases with Crimean-Congo hemorrhagic fever occur almost every year. The most endemic region is the northeast part of the country, such in Kukes and Has. Crimean-Congo hemorrhagic fever (CCHF) is an arboviral zoonotic infection which is endemic in some areas of the country. The aim of this study was to assess the seroprevalence of CCHFV in previous and recent endemic areas of the country. This crosssectional serologic study was conducted in period 2010-2013-2014 by the Institute of Public Health in Tirana, Albania. The survey included 121 sera specimen of cattle which were randomly collected from two districts in Albania. All samples were collected from two districts in Albania and were examined for anti-CCHFV IgG. Of the total of 121 samples examined, 97 (75.7\%) were positive to anti-IgG with ELISA test. The highest positivity rate was among cows (88.3\%) and in recent endemic areas of Kukes and Has. In recent endemic areas humans can potentially contract the disease as compared to other areas of the country.
\end{abstract}

Keywords: Crimean-Congo hemorrhagic fever, domestic animals, Serological Survey

\section{Introduction}

Crimean-Congo haemorrhagic fever (CCHF) is among the most frequent diseases of tick-borne viral origin that it is spread and infects individuals in many parts of the world, in as many as 30 countries in every continent. However, in some parts as Middle East. Africa, Asia and Eastern Europe the disease has a continuously low incidence over several decades. (1). The geographic dissemination of CCHF harmonizes with that of ixodid ticks, especially those of the class Hyalomma. In Europe, Hyalomma marginatum is the principle CCHFV vector, while Hyalomma asiaticum seems, by all accounts, to be the foremost vector in Asia. In 2006, H. 
marginatum was recognized without precedent for the Netherlands and in southern Germany $(2,3)$. Ixodid ticks, particularly those of the family Hyalomma, are both a repository and a vector for the infection. The CCHF infection (CCHFV), a Nairovirus of the family Bunyaviridae, has been detached from 30 types of ixodid tick (3). Various household and wild creatures, for example, dairy cattle, goats, sheep, little well evolved creatures, rodents, and winged animals, in which the disease is for the most part asymptomatic, fill in as opening up has for the infection (4). There is no particular treatment or antibody against CCHF and it is viewed as a developing arboviral zoonotic illness in numerous nations, potentially because of expanded vector bionomics and environmental change. As of late, the frequency of CCHF has expanded quickly in the nations of the World Wellbeing Association Eastern Mediterranean Locale (WHO EMR), with sporadic human cases and flare-ups of CCHF being accounted for from various nations in the district. Notwithstanding the quickly developing rate of the malady, there are right now no precise information on the weight of the sickness in the locale because of the distinctive reconnaissance frameworks for CCHF utilized as a part of these nations. Also, there is no authoritative preventive and control methodology for CCHF inferable from the way that numerous parts of the malady, for example, the support and transmission of the infection and the pathogenesis of the ailment in people, remain ineffectively comprehended (5). Considering the zoonotic nature and public health importance the aim of this study was to assess the seroprevalence of CCHFV in previous and recent endemic areas of the country.

\section{Material and Methods}

\subsection{Sera from cow, sheep and goats}

Blood specimen collection: The survey included 121 sera specimen of cattle which were collected from two districts in Albania over the period 2010-2013-2014. These localities include (Has, Kukes,). Sterile vacutainers were used to draw the blood from jugular veins and were left to clot. The sera were immediately taken to the laboratory and their serum was separated by centrifugation at $3500 \mathrm{rpm}$ for 10 minutes. Each blood sample was stored at $-20^{\circ} \mathrm{C}$ in the Institute of Public Health, Tirana, Albana, until analysis.

\subsection{Indirect ELISA}

Laboratory examination: Adaptation of a human commercial ELISA test for detection of Crimea Congo Hemoorhagic fever (CCHF) antibodies.

The sera collected from domestic animals were tested by an adapted commercial ELISA test which was used for detection of antibodies to CCHFV in human sera (6).

\section{Results \& Discussion}


Distribution of domestic animals by district is shown in table 1 . As noted, most of domestic animals belong to district of Kukes (58.2\%) followed by district of Has $(41.8 \%)$ which are recent endemic areas, with a significant difference as compared to other district $(\mathrm{p}<0.01)$.

Cows account for $50.7 \%$ of animals, followed by sheep (29.6), and goats (2.6\%), $(\mathrm{p}<0.01)$.

Table 1. Distribution of domestic animals by district

\begin{tabular}{|l|l|l|l|l|}
\hline \multirow{2}{*}{ District } & \multicolumn{3}{|l|}{ Type of domestic animals } & Total n (\%) \\
\cline { 2 - 5 } & Cow & Goat & Sheep & \\
\hline Has & 58 & 0 & 0 & $58(58.2 \%)$ \\
\hline Kukes & 18 & 4 & 20 & $42(41.8 \%)$ \\
\hline Total n (\%) & $76(50.7 \%)$ & $4(2.6 \%)$ & $20(29.6 \%)$ & 100 \\
\hline
\end{tabular}

The serologic results are shown in table 2 .

According to ELISA serology the majority of specimens (75.7\%) resulted positive for IgG to CCHF [95\%CI 74.1 - 85.5] (21.9) resulted negative for IgG to CCHF [95\%CI 18.9 - 37.3] . A very small number of specimens (2.4\%) resulted cut-off.

Table 2. ELISA IgG serologic results

\begin{tabular}{|l|l|l|l|}
\hline ELISA IgG & $\mathrm{N}$ & $\%$ & $95 \% \mathrm{CI}$ \\
\hline Cut-off & 6 & $2.4 . \%$ & $1.42-7.48$ \\
\hline Negative & 18 & $21.9 \%$ & $18.9-37.3$ \\
\hline Positive & 97 & $75.7 \%$ & $74.1-85.5$ \\
\hline Total & 121 & $100.0 \%$ & \\
\hline
\end{tabular}

Table 3 presents the serologic result by type of animal. The highest positivity rate was among cows (88.3\%), followed by sheep (95.6\%), and only one case among goats (25\%), $\mathrm{p}<0.01$.

Table 3. Serologic result by type of animal

\begin{tabular}{|l|l|l|l|l|l|}
\hline $\begin{array}{l}\text { Domestic } \\
\text { animals }\end{array}$ & $\begin{array}{l}\text { Total } \\
\text { animal } \\
\text { tested }\end{array}$ & $\begin{array}{l}\text { Cut- } \\
\text { off }\end{array}$ & Negative & Positive & $\begin{array}{l}\text { Prevalence (\%) of } \\
\text { positive specimens }\end{array}$ \\
\hline Cow & 93 & 3 & 15 & 75 & $75(88.3 \%)$ \\
\hline Goat & 4 & 0 & 3 & 1 & $1(25 \%)$ \\
\hline Sheep & 24 & 1 & 3 & 20 & $20(95.6 \%)$ \\
\hline
\end{tabular}




\section{Results Obtained from Indirect ELISA Assay 2010 according to animals, Kukesi prefecture.}
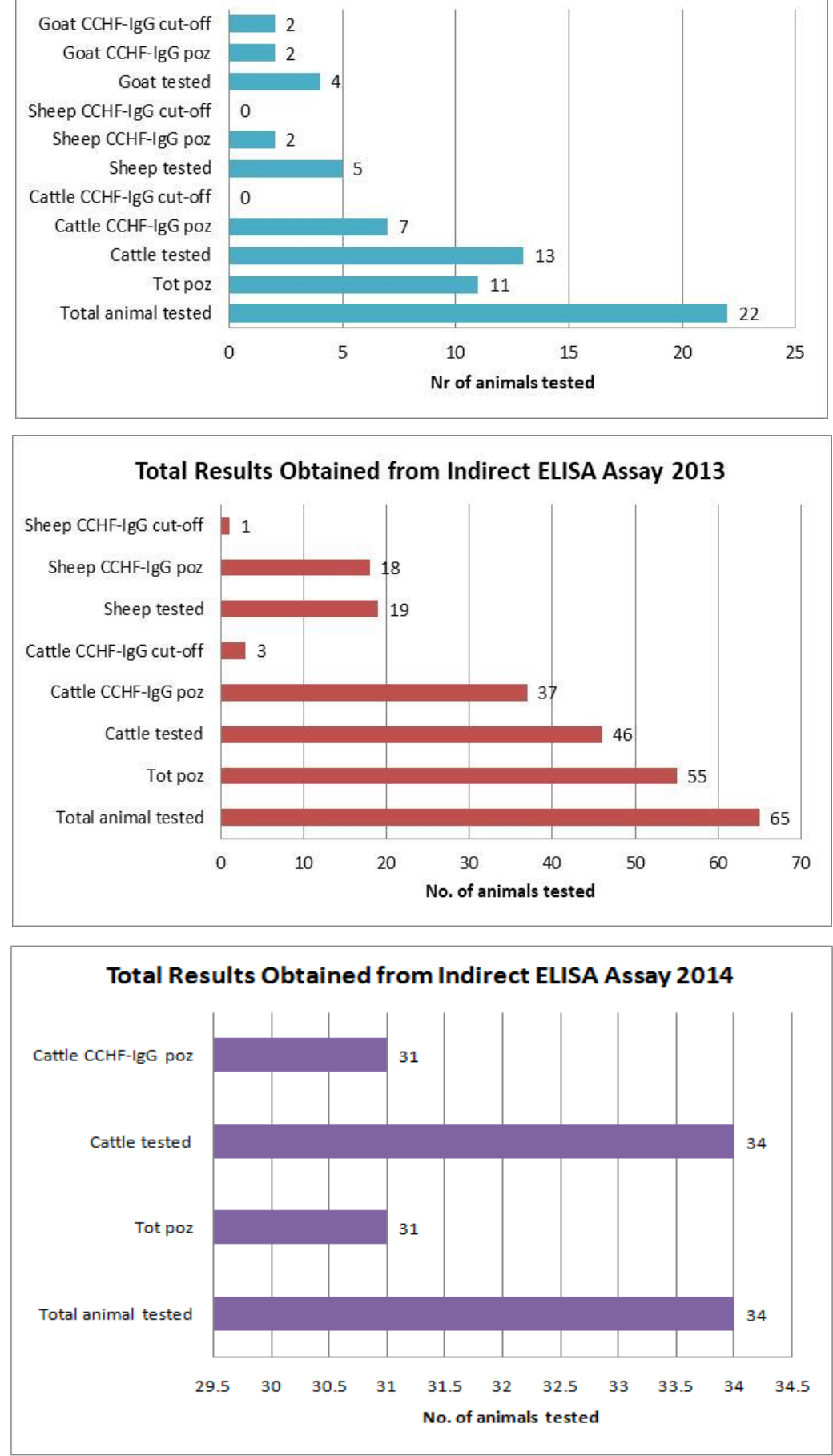
As noted from the above table the endemic areas of Has and Kukes show the highest positivity rate, $58.2 . \%$ and $41.8 \%$ respectively.

Crimean-Congo hemorrhagic fever (CCHF) is a well-known infectious nosology in Albania since its first report in 1974; however, CCHF primary serological studies have been commenced since 1969. For this cross sectional study, we have tested the blood samples of previous and recent endemic areas of the country.

From our outcomes we have diverse qualities in various zones. We found the nearness of disease (antibodies) in creatures in zones where cases with hemorrhagic fever were watched more than one to two decades back, where from that point forward has not been watched cases with hemorrhagic fever in people. This marvel is seen in regions other than Has and Kukes. This demonstrates the disease in these zones is as yet present and we figure it can wind up dynamic. We believe that these outcomes ought to be a flag particularly for human administration which should take solid perception in these territories and in dubious cases ought to instantly take fitting measures. The consequences of our investigation are comparative with different examinations directed in Albania in regards to the seroprevalence of CCHFV in dairy cattle $(6,7,8)$. Countries like Turkey and other Balkan countries except for Greece have recorded flow of CCHF strains among creature hosts, ticks, and people, and have set up CCHF endemicity (10). The wide flare-ups that happened in Turkey were gone before by a very long while of serologic proof of a zoonotic CCHF center. Domesticated animals started from endemic zones came about $14 \%$ seropositive on CCHF infection, just sheep were $32.6 \%$ seropositive (11).

The foundation and support of a CCHF endemic concentration requires a situation supporting an effective contact between able ticks and creature has with moderately high predominance of disease. Heterogeneity in disease levels may happen even inside endemic foci, because of variable atmosphere and ecological reasonableness for ticks and creature has over ongoing decades, adjustments in characteristic biological systems, concentrated farming, an unnatural weather change, and the exponential increment of development of individuals for any reason have given the structure to the extension of a few vector-borne infections all around, from endemic regions to neighboring nonendemic territories and also to far off landmasses. In animal that were infected the virus was amplified to a high level which was sufficiently enough to transmit to the tick vectors, as shown in former investigational surveys which employed cattle inoculation. Seroconversion was detected among infected cattle due to development of a viremia of a low-tire (12).

In the phase of viremia cattle shed the virus and vulnerable humans can contract the disease through bites of infected ticks (13). The outcome of these studies highlight the significant contribution of cattle in the transmission of the infection.

This finding suggested that cattle may play an important role in the epidemiology of the disease. On the contrary, a high rate of lethality from CCHFV infection is observed among humans which reaches up to $30 \%$. 


\section{Conclusions}

The results of this survey suggest that in recent endemic areas humans can potentially contract the disease as compared to other areas of the country. The results demonstrate a widespread infection among cows, sheep and goats with the CCHF virus.Occupational exposure risk should be reduced among professionals handling with animals by implementing the measures of protection and avoiding risky behaviors (14). Doctors and other medical staff ought to think about this infection in individuals with signs and symptoms similar to CCHFV. Mapping of ticks population in the country and promotion are of paramount importance to prevent future CCHF outbreaks.

\section{References}

[1] World Health Organization. (2013). Crimean-Congo haemorrhagic fever (CCHF) in Pakistan. [Online] Available:

http://www.emro.who.int/pandemic-epidemic-diseases/news/cchfpakistan-june-2013.html (June 14, 2013).

[2] Maltezou, H. C., \& Papa, A. (2010). Crimean-Congo hemorrhagic fever: risk for emergence of new endemic foci in Europe? Travel Med Infect Dis, 8, 139143.

[3] Kampen, H., Poltz, W., Hartelt, K., Wölfel, R., \& Faulde, M. (2007). Detection of a questing Hyalomma marginatum marginatum adult female (Acari, Ixodidae) in southern Germany. Exp Appl Acarol, 43, 227-231.

[4] Al-Abri, S. S., Al Abaidani, I., Fazlalipour, M., Mostafavi, E., Leblebicioglu, H., ... \& Nguyen, T. M. N. (2017). Current status of Crimean-Congo haemorrhagic fever in the World Health Organization Eastern Mediterranean Region: issues, challenges, and future directions. Int J Infect Dis, 58, 82-89.

[5] Messina, J. P., Pigott, D. M., Golding, N., Duda, K. A., Brownstein, J. S., Weiss, D. J., \& Nuttall, P. A. (2015). The global distribution of Crimean-Congo hemorrhagic fever. Trans R Soc Trop Med Hyg, 109, 503-513.

[6] Mertens $M$ et al. (2015) Circulation of Crimean-Congo hemorrhagic fever virus in the former Yugoslav Republic of Macedonia revealed by screening of cattle sera using a novel enzyme-linked immunosorbent assay.

[7] Lugaj, A., Koni, M., Schuster, I., Mertens, M., Groschup, M. H., \& Berxholi, K. (2014b). A seroepidemiological survey of Crimean-Congo hemorrhagic fever virus among goats and sheep in Lezhe-Torovica Province, Albania. Albanian J Agric Sci, 13, 28-31.

[8] Lugaj, A., Mertens, M., Groschup, M. H., \& Bërxholi, K. (2014c). Serological survey of CCHFV in cattle in 10 regions of Albania. Int J Res Appl Nat Soc Sci, 2, 55-60. 
[9] Humolli, I., Dedushaj, I., Zupanac, T. A., \& Muçaj, S. (2010). Epidemiological, serological and herd immunity of Crimean-Congo haemorrhagic fever in Kosovo. Med Arh, 64, 91-93.

[10] Dowall, S. D., Richards, K. S., Graham, V. A., Chamberlain, J., \& Hewson, R. (2012). Development of an indirect ELISA method for the parallel measurement of IgG and IgM antibodies against Crimean-Congo haemorrhagic fever (CCHF) virus using recombinant nucleoprotein as antigen. J Virol Methods, 179, 335-341.

[11] Leblebicioglu, H., Ozaras, R., Irmak, H., \& Sencan, I. (2016). Crimean-Congo hemorrhagic fever in Turkey: current status and future challenges. Antiviral Res, 126, 21-34.

[12] Mohamed, M., Said, A., Murad, M., \& Graham, R. (2008). A serological survey of Crimean Congo hemorrhagic fever in animals in the Sharkia Governorate of Egypt. Vet Ital, 44, 513-517.

[13] Swanepoel, R., Shepherd, A. J., Leman, P. A., Shepherd, S. P., \& Miller, G. B. (1985). A common-source outbreak of Crimean-Congo haemorrhagic fever on a dairy farm. S Afr Med J, 68, 635-637.

[14] Abu Salma, A. A. (1995). Meat hygiene in the Sudan: Public health implications of edible offals. MVSc thesis: Faculty of Veterinary Medicine, University of Khartoum, Sudan. 